\title{
ON LOCAL SOLVABILITY OF LINEAR PARTIAL DIFFERENTIAL EQUATIONS
}

\author{
BY FRANÇOIS TREVES
}

The title indicates more or less what the talk is going to be about. It is going to be about the problem which is probably the most primitive in partial differential equations theory, namely to know whether an equation does, or does not, have a solution. Even this is meant in the most primitive terms. I would like to begin by explaining what the terms are.

As you all know, the really difficult analysis these days, and perhaps always, is the global analysis. Well, the problem that I am going to discuss is purely local-in the strictest possible sense: we would like to find out if a linear partial differential equation, with coefficients as smooth as you wish, admits locally a solution. Obviously, in this connection, negative results are very important: and negative results about local solvability have global implications. But of course positive results have also their importance. Let us state precisely what is the problem. The partial differential equation under study will be

$$
P u=f
$$

and we would like to know whether for given $f$, defined in the neighborhood of some point, there are solutions $u$. This is really too vague, so that the first thing we shall do is to make it a little more precise. Let us say that the differential operator $P$ is defined in an open set $\Omega$ of the Euclidean space $R^{n}$ and that we wish to solve the equation in $\Omega$; suppose that the right-hand side $f$ is very regular, say $f \in \mathcal{C}^{\infty}(\Omega)$. Then you would like to know whether there are solutions (defined in $\Omega$ ). Now, the experience we have acquired since 1950 in the field of linear partial differential equations tells us that if you pose the problem in this way, you will encounter very serious difficulties of global nature. After all, $\Omega$ is a manifold, in general it possesses a boundary, or points at infinity, and the fact is that the behaviour

An address delivered before the Cincinnati meeting of the Society by invitation of the Committee to Select Hour Speakers for Western Sectional Meetings, April 19, 1969; received by the editors December 3, 1969.

AMS Subject Classifications. Primary 3501, 3520.

Key Words and Phrases. Partial differential equations, local solvability, distribution, infinitely differentiable functions, compact support, Cauchy problem, analytic functionals, pseudodifferential operators. 
of $f$ at the boundary, or at infinity, may have an influence on the answer to our question. We want to avoid this, for this is much too difficult a problem for us to handle at the present time. We shall therefore assume that $f$ has the best possible behaviour at the boundary, which means vanishes identically in the neighborhood of the boundary. In other words, $f$ has compact support in $\Omega$. Let me point out that local solvability theorems have been known for a long time. The most famous of them, which even the algebraists know, is the theorem of Cauchy-Kovalevska. If $\Omega$ is small enough, if $P$ has analytic coefficients, if $f$ is analytic, and if the standard conditions on characteristics are satisfied, then there always is an analytic solution. The kind of data I shall consider are of a completely different nature: simply by requiring that they have compact support, I exclude the analytic case. In a sense one considers that the analytic case is separate and we want to study the $\mathfrak{e}^{\infty}$ case. In summary we try to solve the equation $P u=f$ for arbitrary right-hand sides $f$, $\mathbb{C}^{\infty}$ with compact support in $\Omega$. Now the question is as to what kind of solution I am willing to consider. Well, to start with, I would like to have the best kind, say $\mathfrak{C}^{\infty}$ solutions. But this turns out to be a difficult request to satisfy, at least for the time being-so that we shall ask for any solution at all, were it to be of the worst kind. What are the worst possible solutions? This depends on your viewpoint. In today's lecture I will consider that distributions are bad enough. Thus we shall seek solutions which are distributions in $\Omega$. Let me mention that you could substantially enlarge the inventory of possible solutions, for instance by allowing what are called Satto hyperfunctions or, when the coefficients of the differential operator $P$ are analytic, by considering analytic functionals. Later on, I hope to say a few words about these aspects.

We have not gone, yet, far enough along the road to simplification: one further step remains to be taken. For the problem, as we have stated it so far, is not local enough. The set $\Omega$ has been kept fixed, and this is too rigid. We shall allow ourselves to choose $\Omega$ at willprovided that it ranges over the collection of open neighborhoods of a given point $x_{0}$. We consider a point $x_{0}$ of $R^{n}$ and we ask the following question: Is there an open set $\Omega$ containing $x_{0}$ such that, given any function $f \in \mathcal{C}^{\infty}$ with compact support contained in $\Omega$, there is a distribution $u$ in $\Omega$ satisfying the partial differential equation $P u=f$ in $\Omega$ ? If the answer to this question is yes, we shall say that the equation $P u=f$ is locally solvable at $x_{0}$. The problem is then to find, if possible, necessary and sufficient conditions in order that a given linear partial differential equation be locally solvable at a given point. 
Now that I have stated the problem, let me say that the talk will mostly be historical. But some of it will be very recent history, because in the last four months the problem, or at least an important part of it, has been nearing complete solution, and it is the progress towards its solution that I would like to describe.

The problem discussed here originated after Hans Lewy, ca. 1956, exhibited his now famous example of a linear partial differential equation in the three-dimensional space which is not locally solvable at any point of $R^{3}$. Lewy's operator is

$$
L=\frac{\partial}{\partial x_{1}}+i \frac{\partial}{\partial x_{2}}+i\left(x_{1}+i x_{2}\right) \frac{\partial}{\partial x_{3}} .
$$

(Allow me to insert a personal anecdote: in $1955 \mathrm{I}$ was given the following thesis problem: prove that every linear partial differential equation with smooth coefficients, not vanishing identically at some point, is locally solvable at that point. My thesis director was, and still is, a leading analyst; his suggestion simply shows that, at that time, nobody had any inkling of the structure underlying the local solvability problem, as it is now gradually revealed.)

Before Lewy's example had been discovered, there were many positive cases which were known. From the viewpoint of later history, the most interesting was presented in Hörmander's thesis (1955); I shall describe it soon. But even prior to this, there were many known cases of local solvability. Let me describe rapidly what was known, on the subject of local solvability, prior to Hörmander. First of all, every equation belonging to any one of the three "classical types" was known to be locally solvable at any point of its domain of definition. By the classical types I mean the elliptic equations, like Laplace's, the parabolic ones, like the heat equation, the hyperbolic, like the wave equation. For decades the study of partial differential equations had been essentially limited to these, and very nice and very important results about them had been obtained. In particular, as I have said, they all are locally solvable at any point. It is worth underlining that the solvability is (in general) strictly local: in general, elliptic equations are not globally solvable; parabolic and hyperbolic equations, under reasonable hypotheses, fare slightly better and are usually solvable in every open set with compact closure.

There was another important class of linear partial differential equations for which solvability was known, as a matter of fact, solvability in any bounded open subset of the Euclidean space, and these were the equations with constant coefficients. In particular they are 
all locally solvable. Thus the relevant case, in the study of local solvability, is the case of variable coefficients. Of course, you realize that local solvability is an intrinsic property, coordinates independent, whereas the property of having constant coefficients depends on the coordinates in which the differential operator is expressed. If you change the coordinates, the coefficients will not usually remain constant.

We come now to the "positive" local solvability theorem in Hörmander's thesis. Consider a linear partial differential operator in $n$ variables, which I must, for the first time, write down explicitly:

$$
P(x, D)=\sum_{|\alpha| \leq m} c_{\alpha}(x) D^{\alpha},
$$

where I have used the standard notation: $\alpha=\left(\alpha_{1}, \cdots, \alpha_{n}\right),|\alpha|=$ $\alpha_{1}+\cdots+\alpha_{n}$,

$$
D^{\alpha}=D_{1}^{\alpha_{1}} \cdots D_{n}^{\alpha_{n}}, \quad D_{j}=-\sqrt{ }(-1) \partial / \partial x_{j} \quad(j=1, \cdots, n) .
$$

The coefficients $c_{\alpha}(x)$ are complex-valued $\mathfrak{e}^{\infty}$ functions (in fact, Hörmander's result is true under much weaker regularity assumptions).

The condition in Hörmander's theorem bears only on the principal part of the operator, i.e., on its leading terms: today one would say on its principal symbol:

$$
P_{m}(x, \xi)=\sum_{|\alpha|=m} c_{\alpha}(x) \xi_{1}^{\alpha_{1}} \cdots \xi_{n}^{\alpha_{n}} \quad\left(x \in \Omega, \xi \in R^{n}, \xi \neq 0\right) .
$$

This is a homogeneous polynomial of degree $m$ with respect to the variables $\xi$ (with coefficients depending on $x$ ).

It has been a standard practice, in the study of linear partial differential equations, to drop the lower order terms. But when is it permitted to do so? To this question there is a kind of philosophical answer, it is not a theorem but more like a conjecture that is to be checked in each instance: you may neglect the lower order terms whenever the real characteristics are simple. This means that the cone of zeros of the equation

$$
P_{m}(x, \xi)=0
$$

(called the characteristic equation) has no singularities in $R^{n}$ (we exclude the origin: at any rate the proper set-up for studying (2) is the 
projective space). This can be rephrased by saying that, for all $\xi \in R^{n}, \xi \neq 0$,

$$
\operatorname{grad}_{\xi} P_{m}(x, \xi) \neq 0 .
$$

Indeed, by Euler's homogeneity formula, whenever $\operatorname{grad}_{\xi} P_{m}(x, \xi)$ vanishes so does $P_{m}(x, \xi)$ and such a zero is perforce multiple. In all this $x$ is kept fixed (but arbitrary).

There are cases where you cannot drop the lower order terms. The best known examples are the heat operator

$$
\partial^{2} / \partial x^{2}-\partial / \partial t
$$

and the Schrödinger operator

$$
\partial^{2} / \partial x^{2}-(1 / i)(\partial / \partial t) .
$$

In both cases the principal symbol is $\xi^{2}$; the characteristics are double. It is clear that consideration of the principal symbol alone will not give you sufficient information: for instance it will not enable you to distinguish between the heat equation and Schrödinger's. It is worthwhile pointing out, however, that consideration of the principal symbol or of some symbol which can be called principal (by means of a modified homogeneity) has been an almost universal practice in the study of PDE's. This is true about the heat and the Schrödinger equations: it is only that one replaces the homogeneity with respect to all variables (as manifested in $P_{m}(x, \xi)$ ) by a separate homogeneity, e.g., of degree 2 in $x$ and degree 1 in $t$ as in (3) and (4). Keeping this in mind one sees easily that most results about linear PDE's are obtained by dropping the lower order terms. One important exception is the theory of linear PDE's with constant coefficients (and there are a few more, of lesser importance).

It should also be pointed out that the principal symbol $P_{m}(x, \xi)$ is a kind of "invariant" attached to the equation (1): it is coordinates invariant in the sense that it is a well-defined function on the cotangent bundle. It is the only simple "invariant" attached to the equation (if one excepts the zero-order terms), the others are more complicated and have not yet been much used, to my knowledge.

Let us return to the solvability result in Hörmander's thesis. As I said the hypotheses concerned only the principal symbol. In keeping with the general principle stated above, the first assumption was that the real characteristics, if there were any, be simple. Hörmander put a label on differential operators which have this property, he said they are of principal type; the label has stuck. The second assumption was, in a sense, more strange: the coefficients $c_{\alpha}(x)$ in the principal part 
(hence, $|\alpha|=m$ ) had to be real. Under these two hypotheses, there is local solvability -at any point in whose neighborhood they hold.

The solvability is strictly local; the exceptions to global solvability are trivial: the simplest is probably the rotation operator in $R^{2} \backslash\{0\}$,

$$
x_{1}\left(\partial / \partial x_{2}\right)-x_{2}\left(\partial / \partial x_{1}\right) .
$$

It satisfies all of Hörmander's conditions, but it is certainly not globally solvable in any annular domain surrounding the origin.

Now, if we go back to Lewy's operator, we see that it has first order. Any first order operator whose principal part does not vanish identically at any point has simple real characteristics, since the gradient with respect to $\xi$ of its principal symbol is constant with respect to $\xi$. Of course, the coefficients in Lewy's operator are not real: otherwise there would be a contradiction. On the other hand, it comes very close to having constantcoefficients. Remember, however, that constant coefficients PDE's are locally (even globally) solvable. In Lewy's operator all coefficients are constant except one, and that one is linear in $x_{1}, x_{2}$. Note also that it is closely related to the CauchyRiemann operator-which, of course, is solvable. In a sense, Lewy's operator is barely on the edge of nonsolvability. But on the other hand, it has that remarkable property of not being locally solvable at any point.

The negative result of Lewy and the positive one of Hörmander, mentioned above, called for some kind of general explanation. The beginning of such an explanation was provided by Hörmander in 1959 with a necessary condition of local solvability. This work, as much as the work of Lewy, is crucial for the subsequent history-for two reasons. First of all, the nature of the necessary condition, found by Hörmander, is remarkable and opens the way to much further investigation. Second, the techniques used in its proof were very original, very striking, and are still the essential techniques one uses, with some improvements and modifications. In the necessary and sufficient conditions known today, the proof of the necessity follows the pattern given by Hörmander in 1959.

Let me state the condition. Clearly, both the positive result of 1955, which required the coefficients of the principal part to be real, and Lewy's example show that the nonreal nature of these coefficients is essential if we are to have nonsolvability. Let me add that the proof of the positive result used a priori estimates in the space $L^{2}$; these could be established because one had control of the commutator $\left[P_{m}(x, D), \bar{P}_{m}(x, D)\right]=P_{m}(x, D) \bar{P}_{m}(x, D)-\bar{P}_{m}(x, D) P_{m}(x, D)\left(\bar{P}_{m}(x, \xi)\right.$ is the homogeneous polynomial of degree $m$ in $\xi$ obtaining by replac- 
ing each coefficient of $P_{m}(x, \xi)$ by its complex conjugate). When the coefficients of $P_{m}$ are real, the above commutator is identically zero, but later work of Hörmander, concerning what he called principally normal equations, confirmed the impression that control of the commutator leads to very good $L^{2}$ estimates. Now, $\left[P_{m}(x, D), \bar{P}_{m}(x, D)\right]$ is a differential operator of order $2 m-1$; familiarity with the derivation of the estimates and the fact that the real characteristics of $P$ are simple (which enables one to neglect lower order terms) strongly suggest that what is relevant, in the commutator, is its principal part, $C_{2 m-1}(x, D)$. Indeed, Hörmander's necessary condition states that if $P$ is locally solvable at the point $x$, then, for all $\xi \in R_{n}, \xi \neq 0$,

$$
P_{m}(x, \xi)=0 \Rightarrow C_{2 m-1}(x, \xi)=0 .
$$

This is the necessary condition of local solvability. When one encounters it for the first time, I am certain that it must seem almost as mysterious as Lewy's example. Of course, it is not satisfied, at any point $x=\left(x^{1}, x^{2}, x^{3}\right)$, by Lewy's operator: for it was the behaviour of this operator that Hörmander wanted to "explain." The simplest operator which does not satisfy it (at every point of the line $x^{1}=0$ ) is

$$
\partial / \partial x^{1}+\sqrt{ }(-1) x^{1}\left(\partial / \partial x^{2}\right)
$$

(for points where $x^{1} \neq 0$, (5), viewed as an operator in $R^{2}$, is elliptic, hence locally solvable).

In a sense, Hörmander's necessary condition is not really satisfactory: for one thing, it lacks a conceptual basis; for another, one would like to know how far it is from being sufficient. At any rate, the next natural step was to seek necessary and sufficient conditions for local solvability. In 1962 Louis Nirenberg and I decided to tackle this problem; it looked rather formidable and we set ourselves very modest goals. We decided to look at a single first order linear partial differential equation, at least as a beginning. Note that such an equation will necessarily have simple characteristics-provided that the coefficients of its principal part do not vanish simultaneously at some point.

We had a starting point, namely Hörmander's necessary condition, exemplified, in particularly simple form, by (5). That condition led quite naturally to studying the commutators, not only the first one, $\left[L_{0}, \bar{L}_{0}\right]$ (I am now denoting by $L_{0}$ the principal part of the first order linear partial differential operator under study), but all the successive ones,

$$
\left[L_{0},\left[L_{0}, \bar{L}_{0}\right]\right], \quad\left[L_{0},\left[L_{0},\left[L_{0}, \bar{L}_{0}\right]\right]\right] \text {, etc., }
$$

and also, perhaps, those where a number of $L_{0}$ have been replaced by 
$\bar{L}_{0}$. Whatever conjecture one could come up to, it could always be tested on the operators

$$
\frac{\partial}{\partial x^{1}}+\sqrt{ }(-1)\left(x^{1}\right)^{k} \frac{\partial}{\partial x^{2}}, \quad k=0,1,2, \cdots
$$

What did we know about these operators? When $k=0$, it is essentially the Cauchy-Riemann operator, everywhere solvable; when $k=1$, we get (5), which is not solvable in any open set intersecting the line $x^{1}=0$. Of course, when $k>1$, Hörmander's necessary condition would not provide any more information. But an easy modification of its proof shows that (6) is not solvable in any such open set when $k$ is $o d d$. On the other hand, when $k$ is even, (6) is locally solvable (at any point) - for a very simple reason: namely that one can write explicitly, by a modification of Cauchy's formula, a solution to the corresponding equation (1). I will give, a little later, a different proof of this fact, a proof which ought to throw some light, I hope, on the question of solvability.

The facts known about (6) raise the question of the relation between the number $k$ and the various commutators of $L_{0}$ and $\bar{L}_{0}$. It is convenient, at this point, to modify a little bit our approach and instead of looking at the complex vector fields $L_{0}$ and $\bar{L}_{0}$, to look at the real vector fields $A=\operatorname{Re} L_{0}, B=\operatorname{Im} L_{0}$. In the case of (6),

$$
A=\partial / \partial x^{1}, \quad B=\left(x^{1}\right)^{k} \partial / \partial x^{2} .
$$

Let us then set $C_{1}=[A, B], C_{p+1}=\left[A, C_{p}\right](p=1,2, \cdots)$; these are real vector fields; let us denote by $C_{p}(x, \xi)$ the symbol of $C_{p}$. In the case of (6),

$$
\begin{aligned}
C_{p}(x, \xi) & =\frac{k !}{(k-p) !}\left(x^{1}\right)^{k-p}\left(i \xi_{2}\right) & & \text { if } p \leqq k, \\
& =0 & & \text { if } p>k .
\end{aligned}
$$

Consider a point $(x, \xi)$ such that $x^{1}=0, \xi_{1}=0, \xi_{2} \neq 0$. At any such point, the symbols of $A, B$ and of every $C_{p}$ for $p<k$ vanish; $C_{k}(x, \xi)$ does not. This provides a characterization of the integer $k$ which seems not to depend on the peculiarities of the example (6). The concept can easily be extended to higher order equations. Indeed, let $A(x, D)$ (resp. $B(x, D)$ ) denote the real (resp. imaginary) part of $P_{m}(x, D)$. We may form the successive commutators or, rather, the principal parts of these commutators:

$$
\begin{aligned}
& C_{1}(x, D)=\text { principal part of }[A(x, D), B(x, D)], \\
& C_{p}(x, D)=\text { principal part of }\left[A(x, D), C_{p-1}(x, D)\right] \quad \text { for } p>1 .
\end{aligned}
$$


We look now at the symbols of all these operators, in $(x, \xi)$-space ( $\xi$ must always be different from zero), and with every point $(x, \xi)$ we associate a number $k_{0}(x, \xi):$ this is the least integer $k$ such that $C_{k}(x, \xi) \neq 0$. We agree that $k_{0}(x, \xi)$ is taken to be zero whenever $P_{m}(x, \xi) \neq 0$. Then, in analogy with what happens in the case (6), we shall conjecture that the parity of all these numbers $k_{0}(x, \xi)$ determines whether there is local solvability in the (open) set where the point $x$ ranges.

Well, this approach cannot possibly lead to the full answer. Indeed it has several serious defects: for one, it assigns a privileged role to the real part $A(x, D)$, and this is inadmissible, since $A$ and $B$ can be interchanged by multiplying $P_{m}$ by $\sqrt{ }(-1)$; such a multiplication certainly cannot affect solvability. Also the approach will completely fail if all the commutators happen to vanish at some point $(x, \xi)$, as in the example

$$
\partial / \partial x^{1}+\sqrt{ }(-1) \exp \left(-1 /\left(x^{1}\right)^{2}\right) \partial / \partial x^{2} .
$$

There is a way around the latter difficulty; this is a very important observation, which will also allow us to eliminate the first defect we mentioned. It has to do with the most classical part of PDE theory, the part that goes back to Lagrange, Hamilton, Jacobi. When dealing with functions such as $A(x, \xi), B(x, \xi)$, etc., defined (and smooth) in the $(x, \xi)$-space (in the cotangent bundle as we say now), one can introduce the Hamilton-Jacobi equations which, for $A$, e.g., read

$$
\begin{aligned}
& d x / d t=\operatorname{grad}_{\xi} A(x, \xi), \\
& d \xi / d t=-\operatorname{grad}_{x} A(x, \xi) .
\end{aligned}
$$

I am taking $A$ here, but I could as well consider $B, C_{p}$, etc. Note however that in our examples (5), (6), (7) the gradient with respect to $\xi$ of $A(x, \xi)$ did not vanish at any point $(x, \xi)$ under consideration. Our basic hypothesis, that $P$ is of principal type, means that given any point $\left(x_{0}, \xi^{0}\right), \xi^{0} \neq 0$, either $\operatorname{grad}_{\xi} A$ or $\operatorname{grad}_{\xi} B$ does not vanish at that point, or that none does. We may assume that $\operatorname{grad}_{\xi} A\left(x_{0}, \xi^{0}\right) \neq 0$. In this case, the (unique) solution $(x(t), \xi(t))$ of (8) such that $x(0)=x_{0}$, $\xi(0)=\xi^{0}$ describes a true curve in $(x, \xi)$-space whose pro ection on the $x$-space is, in fact, a curve. Let us denote by $\Gamma_{A}\left(x_{0}, \xi^{0}\right)$ the integral curve of (8) through $\left(x_{0}, \xi^{0}\right)$ in the $(x, \xi)$-space; it is of ten called the bicharacteristic strip of $A$ through that point. It follows at once from the form of (8) that the function $A(x, \xi)$ must be constant along such a curve. Thus, if we assume that $A\left(x_{0}, \xi^{0}\right)=0$, we will have $A=0$ on the whole of $\Gamma_{A}\left(x_{0}, \xi^{0}\right)$. In this case we shall refer to $\Gamma_{A}\left(x_{0}, \xi^{0}\right)$ as the null bicharacteristic strip of $A$ through $\left(x_{0}, \xi^{0}\right)$. 
The relevance of these bicharacteristic strips from our viewpoint is due to the following fact: an immediate computation shows that the symbol of the first "commutator" $C_{1}(x, D)$, the principal part of $[A(x, D), B(x, D)]$ is given by

$$
C_{1}(x, \xi)=\sum_{j=1}^{n}\left\{\frac{\partial A}{\partial \xi_{j}}(x, \xi) \frac{\partial B}{\partial x^{j}}(x, \xi)-\frac{\partial A}{\partial x^{j}}(x, \xi) \frac{\partial B}{\partial \xi_{j}}(x, \xi)\right\},
$$

that is,

$$
C_{1}(x, \xi)=(d B / d t)(x, \xi),
$$

where $d / d t$ denotes the differentiation in the direction tangential to the bicharacteristic strip of $A$ through the point $(x, \xi)$. By iteration,

$$
C_{p}=d^{p} B / d t^{p} \text {. }
$$

Let us then suppose that $P_{m}\left(x_{0}, \xi^{0}\right)=0$, that is, that both $A$ and $B$ vanish at $\left(x_{0}, \xi^{0}\right)$. Let us look at the function $B(x, \xi)$ restricted to the null bicharacteristic strip of $A$ through $\left(x_{0}, \xi^{0}\right), \Gamma_{A}\left(x_{0}, \xi^{0}\right)$. Hörmander's necessary condition can be restated by saying that if the equation $P u=f$ is locally solvable at $x_{0}$ then the first derivative of $B$ along $\Gamma_{A}\left(x_{0}, \xi^{0}\right)$ must vanish at $\left(x_{0}, \xi^{0}\right)$. In the case $(6)$ where, say $\xi^{0}=(0,1)$ and $x_{0}^{1}=0$, we see that there is local solvability if the first derivative of $B$ along $\Gamma_{A}\left(x_{0}, \xi^{0}\right)$ which does not vanish is of even order, and that there is no solvability if this derivative is of odd order. But there is a way of rephrasing this property which does not rely on the fact that the zero of $B(x, \xi)$ along $\Gamma_{A}\left(x_{0}, \xi^{0}\right)$ is of finite order, and which therefore enables us to extend the property to equations such as (7): namely, that

(9) $B(x, \xi)$ does not change sign at $\left(x_{0}, \xi^{0}\right)$ along the null bicharacteristic strip of $A(x, \xi)$ through that point.

We could now make the conjecture that local solvability of the equation $P u=f$ in the open set $\Omega$ is equivalent with the fact that (9) is valid for all $x_{0} \in \Omega$ and all $\xi^{0} \in R^{n}, \xi^{0} \neq 0$-if it were not for the fact that the statement (9) is blatantly dissymmetric in $A$ and $B$. We shall therefore symmetrize in the following fashion:

(10) Let $x_{0} \in \Omega, \xi^{0} \in R_{n}, \xi^{\circ} \neq 0$, be such that $P_{m}\left(x_{0}, \xi^{0}\right)=0$. Let $z$ be any complex number such that

$$
\operatorname{grad}_{\xi} \operatorname{Re}\left(z P_{m}\right) \neq 0 \quad \text { at }\left(x_{0}, \xi^{0}\right) \text {. }
$$

Then the function $\operatorname{Im}\left(z P_{m}\right)(x, \xi)$ does not change sign at $\left(x_{0}, \xi^{0}\right)$ along the null bicharacteristic strip of $\operatorname{Re}\left(z P_{m}\right)(x, \xi)$ through this point.

Clearly, solvability of Equation (1) is unchanged if we substitute $z P, z \neq 0$, for $P$. Condition (10) may seem somewhat awkward, as it 
involves all complex numbers $z \neq 0$. But the remarkable fact is that, essentially, ${ }^{1}$ it is true for all numbers $z \neq 0$ as soon as it is true for at least one of them. More precisely:

Theorem 1. Let $q(x, \xi)$ be a complex-valued function of $(x, \xi)$, defined, $\mathrm{e}^{\infty}$ and nowhere vanishing in some open neighborhood of $\left(x_{0}, \xi^{0}\right)$. Let us suppose that neither $\operatorname{grad}_{\xi} A$ nor $\operatorname{grad}_{\xi} \operatorname{Re}\left(q P_{m}\right)$ vanish at $\left(x_{0}, \xi^{0}\right)$. Then, if Property (9) holds, the analogous property, where $P_{m}(x, \xi)$ is replaced by $q(x, \xi) P_{m}(x, \xi)$, also does.

This was proved in the first order case (i.e., $m=1$ ) in [6]; it is proved for arbitrary $m$ in [7] under the additional assumption that any change of sign of $B$ along a null bicharacteristic strip of $A$ must occur at a zero of finite order of $B$ (along that strip). ${ }^{2}$ Let me add that these proofs are by no means trivial.

If we take Theorem 1 into account and keep in mind that, at any point $(x, \xi)$, either $\operatorname{grad}_{\xi} A(x, \xi)$ or $\operatorname{grad}_{\xi} B(x, \xi)$ are $\neq 0$, we see that the validity of Property (10) for all $x_{0} \in \Omega, \xi^{0} \in R_{n}, \xi^{0} \neq 0$, can be rephrased as follows:

(11) $\operatorname{Re} P_{m}(x, \xi)$ does not change sign along any null bicharacteristic of $\operatorname{Im} P_{m}(x, \xi)$ (lying over $\Omega$ ) and $\operatorname{Im} P_{m}(x, \xi)$ does not change sign along any null bicharacteristic strip of $\operatorname{Re} P_{m}(x, \xi)$.

We have tacitly agreed not to speak of a null bicharacteristic strip of a function of $(x, \xi)$ unless the gradient with respect to $\xi$ of this function does not vanish anywhere on the strip.

We may now formulate the main conjecture:

CONJECruRE. The equation $P u=f$ is locally solvable at every point of $\Omega$ if and only if (11) holds.

This is the conjecture which Nirenberg and I were led to make, in 1962. Note that it is compatible with the positive result in Hörmander's thesis, where the coefficients of $P_{m}(x, \xi)$ had to be real, i.e. $\operatorname{Im}\left(i^{m} P_{m}(x, \xi)\right) \equiv 0$. Soon after, we were able to prove that it indeed held for first order differential equation-with a "minor" qualification: the necessity of Condition (11) was established only in the case where, along the null bicharacteristic strips of $A$, the changes of sign of the function $B$ occurred at zeros of finite order (as a matter of fact, we could prove necessity even if some changes of sign occurred at zeros of infinite order, but not in full generality: this is still an open question). This is not to say that $B$ could not have zeros of infinite order, but only that $B$ could not change sign at such a zero; for instance, $B$ could

${ }^{1}$ Not "essentially" but "truly"; see footnote 2.

${ }^{2}$ In [7] Theorem 1 is proved in full generality: the hypothesis about the changes of sign of $B$ is removed. 
very well vanish identically on a null bicharacteristic strip of $A$, as in the operator

$$
\partial / \partial x^{1}+\sqrt{ }(-1) x^{2} \partial / \partial x^{2}
$$

Of course, the case of analytic coefficients was completely settled; in this case, Condition (11) is necessary and sufficient-without any qualification.

This was the state of affairs in 1963. Since then, until recently, nothing much has happened. We could not extend our result to higher order equations. We could not handle the lower order terms. This was due to a certain feature in our treatment of the first order case. I should say that the obstruction was on the side of the sufficiency proof. As far as necessity of (11) was concerned we had the feeling that it was, and would remain within our reach, that all we had to do was, some day, to sit down and muddle through the technicalities, and we would get what we expected. But not so with sufficiency! Recent experience has confirmed that these were sound feelings.

I would like to explain what the obstruction was, for it had an important implication concerning first order equations. The proof of sufficiency was based on an estimate of a quite unusual nature, and we could not see, we still cannot see, how to extend it to higher order equations. Let me describe the estimate we did obtain, and also the one we would have liked to obtain. In fact I shall begin by the latter. Let us denote by $P^{\prime}$ the formal transpose of $P$. This is the operator derived from $P$ by integration by parts:

$$
\int(P u) v d x=\int u\left(P^{\prime} v\right) d x, \quad u, v \in C_{c}^{\infty}(\Omega) .
$$

Now what we would have liked to obtain is an estimate

$$
\int|u|^{2} d x \leqq C \int\left|P^{\prime} u\right|^{2} d x, \quad u \in C_{c}^{\infty}(\Omega)
$$

Here $\Omega$ is some open set. A very elementary lemma about Hilbert spaces (or else a straightforward application of the Hahn-Banach theorem) shows that, if (12) holds, then to every $f \in L^{2}(\Omega)$ there is $u \in L^{2}(\Omega)$ such that $P u=f$ (in $\Omega$, in the distribution sense).

Thus (12) is what one needs if he is to prove solvability in the " $L$ " sense." As a matter of fact, we would be very happy if we could have even more: if we could choose the constant $C$ in (12) so that it decreases and tends to zero together with the diameter of $\Omega$. In such a case one can always perturb the zero order term in $P$ (or in $P^{\prime}$ ) as widely as he wishes, for the smallness of $C$ will take care of that. In 
other words, estimates like (12), with $C \searrow 0$ with diam $\Omega$, are stable under zero order perturbations. This stability is crucial if we are to try to extend such an estimate to higher order equations; transposed into this context it would mean that we could neglect lower order terms.

As I said (12) is not at all what we proved; what we proved was the estimate

$$
\int|u|^{2} d x \leqq C \int\left|\operatorname{grad}\left(P^{\prime} u\right)\right|^{2} d x, \quad u \in C_{c}^{\infty}(\Omega)
$$

Now, here, even when the constant $C \searrow 0$ with diam $\Omega$, one cannot perturb the zero order terms in $P^{\prime}$ and deduce a similar estimate; such a perturbation introduces a quantity of the form

$$
\text { const. } \int|\operatorname{grad} u|^{2} d x
$$

on the right-hand side of (13), and there is no way of absorbing it into the left-hand side. Of course, (13) is valid (under Assumption (11)) regardless of what the zero order terms in $P$ are; but as far as the method of proof is concerned, you cannot neglect these zero order terms, you have to handle them with special tricks. This is what precludes extension to higher order case since we could not possibly hope to extend those tricks so as to handle lower order, but not any more zero order, terms.

Moreover an estimate such as (13) does not imply $L^{2}$ solvability. What it does imply is that, to every $f \in L^{2}(\Omega)$ there is $u \in H^{-1}(\Omega)$ satisfying the equation $P u=f$ in $\Omega ; H^{-1}(\Omega)$ is the (Sobolev) space of distributions in $\Omega$ which can be expressed as finite sums of first order partial derivatives of functions belonging to $L^{2}(\Omega)$.

It is now time to describe more recent results. Last December, 1968, I was able to show that Property (11) is sufficient for local solvability in $\Omega \subset R^{2}$. More precisely:

TheOREM 2. In the case of two independent variables $(n=2)$, if Property (11) holds, the equation $P u=f$ is locally solvable at every point of $\Omega$.

Conversely, suppose that, for some $x_{0} \in \Omega$ the following is true:

(14) there is $\xi^{0} \neq 0$ such that $P_{m}\left(x_{0}, \xi^{0}\right)=0, d_{\xi} \operatorname{Re} P_{m}\left(x_{0}, \xi^{0}\right) \neq 0$, and such that, along the null bicharacteristic strip of $\operatorname{Re} P_{m}$ through $\left(x_{0}, \xi^{0}\right)$, Im $P_{m}$ has a zero of finite odd order at that point.

Then the equation $P u=f$ is not locally solvable at $x_{0}$. 
But now something more is true, for the estimate used to prove the existence of solutions is of the kind (12), not of the kind (13). More precisely, since we deal with a differential operator of order $m$, the estimate reads:

$$
\sum_{|\alpha| \leqq m-1} \int\left|D^{\alpha} u\right|^{2} d x \leqq \epsilon \int\left|P^{\prime} u\right|^{2} d x, \quad u \in C_{*}^{\infty}(\Omega),
$$

where $\epsilon \searrow 0$ when diam $\Omega \searrow 0$. This is optimal-since we know it is optimal for hyperbolic equations (any better estimate, i.e. where derivatives of order $>m-1$ of $u$ would be dominated, would imply hypoellipticity). It is not very difficult to derive from (15) that to every $f \in L^{2}(\Omega)$ there is $u \in H^{m-1}(\Omega)$ such that $P u=f$ in $\Omega ; H^{m-1}(\Omega)$ is the Sobolev space of functions whose distributions derivatives of order $\leqq m-1$ belong to $L^{2}(\Omega)$.

Differential operators in two independent variables, if they are of principal type, are quite special. Indeed, their principal symbol $P_{m}(x, \xi)$ is a polynomial in the two variables $\left(\xi_{1}, \xi_{2}\right)$, homogeneous of degree $m$, hence can be factorized,

$$
P_{m}(x, \xi)=a(x) \prod_{j=1}^{m}\left(\xi_{1}-\lambda_{j}(x) \xi_{2}\right),
$$

and all the properties of $\boldsymbol{P}$ (at least, those of interest to us here) can be translated in terms of the characteristic roots $\lambda_{j}(x)$. These are functions of $x$ alone; $\xi$ does not enter. Because of this, Condition (11) can be rephrased, in this case, in the following manner:

$\left(11^{\prime}\right)$ For each $j=1, \cdots, m$, the function $\operatorname{Im} \lambda_{j}$ does not change sign along the integral curves of the vector field

$$
\partial / \partial x^{1}-\left(\operatorname{Re} \lambda_{j}(x)\right) \partial / \partial x^{2}
$$

Suppose for a moment that the coefficients of $P_{m}(x, \xi)$ are analytic. Then the changes of sign of $\operatorname{Im} \lambda_{j}$ must perforce occur at points belonging to a proper analytic subset of $\Omega$. Even in the $C^{\infty}$ case, they certainly could not occur everywhere. Nonsolvability is therefore a "rare" event. This shows that an example such as Lewy's, where nonsolvability occurs at every point, can only be encountered if the number of independent variables is $>2$.

What is now known when there are $n>2$ independent variables? Well, in the first order case, we have now the best possible result. Indeed, I have been able to prove an estimate of the kind (12), here again with a constant $C \searrow 0$ with $\operatorname{diam} \Omega$. Thus:

Theorem 3. If $\boldsymbol{P}$ is a first order linear partial differential operator 
with $C^{\infty}$ coefficients in $\Omega$, having Property (11), every point $x_{0} \in \Omega$ has an open neighborhood $U\left(x_{0}\right) \subset \Omega$ such that

$$
P L^{2}\left(U\left(x_{0}\right)\right) \supset L^{2}\left(U\left(x_{0}\right)\right) .
$$

This is an improvement on the 1962 result concerning the existence of solutions to first order PDEs. The proof of Theorem 3 uses Theorem 2 via the observation that, locally, any first order linear PDE, of the form ${ }^{3}$

$$
\frac{\partial u}{\partial t}+\sqrt{ }(-1) \sum_{j=1}^{n} b_{j}(x, t) \frac{\partial u}{\partial x^{j}}=f,
$$

having Property (11), can be transformed by a change of variables into a first-order PDE in two independent variables. The unfortunate thing is that such changes of variables are to be performed in the complement of the set

$$
\left\{x ; b_{j}(x, t)=0, j=1, \cdots, n,(x, t) \in \Omega\right\},
$$

and their domain of validity tends to shrink as we approach this set. The derivatives of the cut-off functions which are used tend, in absolute value, to $+\infty$ as the domains of the local transformations shrink and this creates some difficulty when one patches together the $L^{2}$-estimates which have been established in each domain individually. The derivatives must be kept under control and this is achieved by means of a lemma which resembles very much a classical lemma of Whitney used to extend to the whole space differentiable functions defined in a closed set.

At last I come to PDEs of principal type, in any number of independent variables, of any order. Very recently, Louis Nirenberg and I have shown the necessity of Condition (11), under the usual restrictions. Precisely we proved:

Theorem 4. Suppose that for some point $x_{0} \in \Omega$, Property (14) holds. Then the equation $P u=f$ is not locally solvable at $x_{0}$.

As a matter of fact, we prove this theorem when $P$ is a pseudodifferential operator of order $m$ (which is then an arbitrary real number), under the assumption that the homogeneity degrees, with respect to $\xi$, of the various terms in the symbol $P(x, \xi)$ of $P$ are $m, m-1, m-2$,

${ }^{3}$ It is easy to see that every first-order linear PDE with $C^{\infty}$ coefficients, without any zero order term, which does vanish identically at any point, can be brought into that form-at least locally and up to a nowhere vanishing factor-by a change of coordinates. 
$\cdots, m-k, \cdots$. One must then modify the statement of Condition (14) as follows:

$\left(14^{\prime}\right)$ there is $\xi^{0} \neq 0$, such that $P_{m}\left(x_{0}, \xi^{0}\right)=0, d_{\xi} \operatorname{Re} P_{m}\left(x_{0}, \xi^{0}\right) \neq 0$, and such that, along the null bicharacteristic strip of $\operatorname{Re} P_{m}$ through $\left(x_{0}, \xi^{0}\right)$, Im $P_{m}$ has a zero of finite odd order at that point and changes sign there from minus to plus.

This property is invariant under multiplication of $P_{m}(x, \xi)$ by a nowhere vanishing function $q(x, \xi)$ as considered in Theorem 1 . This is shown in [7]. In the pseudodifferential case one must also suitably modify the concept of local solvability.

We have learned by Professor Olga Oleinik that one of her Ph.D. students, Y. V. Egorov, has proved a result which appears to be stronger than Theorem 4 . I must say that I am not extremely surprised that others should have also proved this kind of theorem, as it is really a question of reworking, with a little bit of skill, the original proof of the necessary condition of Hörmander of 1959.

Theorems 2, 3, 4 represent the results known at the present time on the subject of local solvability of linear PDE's in the sense of functions and distributions. ${ }^{4}$

I would like to end this lecture by a few remarks about solvability in a more general sense than function or distribution solvability. As a starting point for these remarks I wish to take a fresh look at the operators (6) which I now write

$$
\partial / \partial t+i t^{k} \partial / \partial x, \quad i=\sqrt{ }-1 .
$$

After performing a Fourier transformation in the variable $x$, the associated equation reads

$$
\hat{u}_{t}+t^{k} \xi \hat{\imath}=\hat{f}, \quad f \in\left(C_{c}^{\infty}\right)_{x, t}
$$

where the "hats" denote Fourier transformation in $x$ (on the Fourier transforms-side the variable is denoted by $\xi$ ). We may solve (16) in two different ways:

$$
\mathfrak{u}(\xi, t)=\int_{-\infty}^{t} \hat{f}(\xi, s) \exp \left(-\frac{t^{k+1}-s^{k+1}}{k+1} \xi\right) d s,
$$

${ }^{4}$ During the summer of $1969, \mathrm{~L}$. Nirenberg and I were able to prove our conjecture (p. 124) for all PDEs of principal type, of any order, in any number of variables, if they have analytic coefficients. The results obtained are the best possible, as we prove an estimate of the kind (15). On the other hand, Yu. V, Egorov claims to have obtained very general sufficient conditions for the validity of the so-called subelliptic estimates, which imply local solvability. But it is not clear to us, at this time, what his conditions are (added on the proofs). 


$$
\hat{u}(\xi, t)=-\int_{t}^{+\infty} \hat{f}(\xi, s) \exp \left(-\frac{t^{k+1}-s^{k+1}}{k+1} \xi\right) d s .
$$

If we want to return to the original equation we must then perform an inverse Fourier transformation, reverting from the variable $\xi$ to $x$. But we will end up with a distribution solution if and only if we start from a solution of (16) which is tempered with respect to $\xi$, that is to say (roughly speaking), which, as a function of $\xi$, does not grow at infinity faster than some polynomial. The question is then whether such a solution of (16) exists. Suppose that $k$ is even. Then we could select (17) as the solution for $\xi>0$ and (18) when $\xi<0$ (the solution does not have to be a continuous function of $\xi$ but only a tempered distribution in $\xi !)$. It is clear that, with these choices, the exponent

$$
-\left(t^{k+1}-s^{k+1}\right) \xi /(k+1)
$$

in each integral, remains bounded. We have thus defined a solution $\hat{\mathrm{u}}$ of (16) which is an $L^{\infty}$ function of $\xi$. But what if $k$ is odd? It is clear that this procedure will not work. By virtue of the nonexistence theorems stated earlier we know that no procedure will work. On the other hand, we have at our disposal lots of solutions of (16), and they have very nice expressions; one feels that they should be useful in some computations at least, and that we should be able to define and use their inverse Fourier transforms. This is indeed the case, but these inverse Fourier transforms will, of course, not be distributions - they will be objects of a fairly general nature, known under the name of analytic functionals. These are linear functionals on spaces of holomorphic functions, exactly like distributions are linear functionals on the space of $C^{\infty}$ functions with compact support. One of the essential differences between the former and the latter is that there exist partitions of unity consisting of $C_{c}^{\infty}$ functions (subordinated to any locally finite open covering of the base space) but there are no such partitions consisting of holomorphic functions. This difference has, of course, extremely deep consequences; one of these is that one can define the support of a distribution but not the support of an analytic functional. One has the right to say that a given distribution takes these values in a given open set and these different values in a different open set, but nothing of the sort may be asserted about analytic functionals. In summary, distributions are localizable whereas analytic functionals are not. This does not mean that the latter are useless, it only means that they do not have the properties which go with localization. As a matter of fact, a great deal can be said, and has been said, about analytic functionals (see e.g. the works 
of A. Martineau, in particular [5]), mainly through their "Fourier" transforms, which happen to be called Fourier-Borel transforms (sometimes Fourier-Laplace, sometimes Borel-Laplace transforms). Let $\Omega$ be an open subset of the complex $n$-dimensional space $C^{n}$ intersecting the real space $R^{n}$ and let $\Omega_{R}=\Omega \cap R^{n}$; then any $L^{1}$ function $f(x)$ with compact support in $\Omega_{R}$ defines a unique analytic functional on $\Omega$ through the formula

$$
\langle f, h\rangle=\int f(x) h(x) d x,
$$

where $h$ is an arbitrary holomorphic function in $\Omega$. Similarly any distribution with compact support in $\Omega_{R}$ defines a unique analytic functional in $\Omega$. Thus distributions with compact support can be identified with certain analytic functionals (but of course not every analytic functional can be thus defined by a distribution with compact support - otherwise our remarks above about localization would be somewhat irrelevant). Keeping this in mind, I would like to state a theorem about solvability in spaces of analytic functionals. In fact, it concerns local solvability of the Cauchy problem posed for a differential operator of order $m$ with analytic coefficients (only analytic coefficients can act as multipliers on analytic functionals). In the present case we must distinguish between space variables, $x=\left(x^{1}, \cdots, x^{n}\right)$, and a time variable; we assume that the differential operator under study has an expression

$$
P=D_{t}^{m}-\sum_{j=1}^{m} c_{j}\left(x, t, D_{x}\right) D_{t}^{m-j},
$$

where each $c_{j}\left(x, t, D_{x}\right)$ is a differential operator of order $\leqq j$ with respect to $x$ with coefficients which are analytic functions of $(x, t)$, say in a neighborhood $U$ of the origin in $R^{n+1}$. We consider the Cauchy problem for $P$ relative to the hyperplane $t=0$, which is the problem of finding a solution $u$ to

$$
\begin{aligned}
P u & =f \quad \text { in } U, \\
D_{t}^{k} u & =g_{k} \quad \text { in } U \cap\{(x, t) ; t=0\}, \quad k=0, \cdots, m-1 .
\end{aligned}
$$

The "objects" $g_{k}$ depend only on $x$, they are called the Cauchy data; $f$ will be a function of $t$, say a continuous function of $t$, valued in a space of analytic functionals with respect to the variables $x$; and we shall also take the $g_{k}$ to be analytic functionals with respect to $x$. Possibly after some shrinking of the neighborhood $U$, it can be (roughly) 
stated that the problem (19)-(20) has a unique solution which is a $C^{1}$ function of $t$ with values in the space of analytic functionals with respect to $x$. For the precise statement, see $[15, \S 11]$. The proof of this theorem is easy, it is a dual form of the Cauchy-Kowalewski theorem. I should add that much more than existence and uniqueness of the solution $u$ can be asserted: the solution $u$ can be expressed in terms of the data, $f$ and the $g_{k}$, by means of an integral formula having a kernel which is an analytic function of the intervening variables. On this subject see [15, Chapter II], also [16]. At any rate, we see that when $f$ and the $g_{k}$ are $C^{\infty}$ functions of $x$ and $t$ with compact support or distributions of $x$ and $t$ with compact support ( $f$ does not have to be a function with respect to $t$ in order that we have a solution $u$ to (19)(20), $f$ can be only a distribution in $t$, but then $u$ will also have to be a distribution in $t$ ), the equation (19) admits a solution-always! but of course it is not going to be a distribution, only a distribution with respect to $t$, but an analytic functional with respect to $x$.

It should be mentioned that we should not expect a theorem such as the one just stated to hold for differential operators with $C^{\infty}$ coefficients-not only because these coefficients would not multiply analytic functionals, for we would be willing to try to replace analytic functionals with something else. The obstruction here is the uniqueness part in the statement: indeed it is known that there is no uniqueness in the (noncharacteristic) Cauchy problem for certain equations with $C^{\infty}$ coefficients-even when the solutions happen to be $C^{\infty}$ functions of $x$ and $t$ with compact support! Examples of such equations have been constructed by Paul Cohen and A. Pliš.

There are objects, that is, generalized functions, which are more general than distributions and, in a certain sense, less general than analytic functionals - and which manage to retain the localization properties: they are the, so-called, Sâto hyperfunctions. Very roughly speaking they are boundary values (carried by pieces of the real space $R^{n}$ ) of holomorphic functions (defined in complex neighborhoods of those "pieces" of $R^{n}$ ); they have attracted the attention of theoretical physicists as they lend themselves to certain operations which are not allowed in distribution theory. Also they have provided solutions to $\mathrm{PDE}$ with constant coefficients in cases where no distribution solution exists (see [1]). Thus it is only natural to ask the question as to whether one has to go so far as analytic functionals in order to attain solvability (at least in the case of equations with analytic coefficients) and whether Sâto hyperfunctions, with their localization properties, would not suffice. Alas, this does not seem to be the case. A student of Laurent Schwartz, Pierre Schapira, has 
shown that, at least for first order PDE with analytic coefficients, nonsolvability in the distribution sense implies nonsolvability in the space of Sâto hyperfunctions (see [11], [12]). I am convinced that what lies at the root of nonsolvability of PDE's of principal type is not our insistence on considering distributions, it is not just compliance with fashion; it is our insistence in having solutions which are localizable generalized functions.

\section{BIBLIOGRAPHY}

1. R. Harvey, Hyperfunctions and linear partial differential equations, Proc. Nat. Acad. Sci. U. S. A. 55 (1966), 1042-1046. MR 34\#495.

2. L. Hörmander, On the theory of general partial differential operators, Acta Math. 94 (1955), 161-248. MR 17, \#853

3. - Differential equations without solutions, Math. Ann. 140 (1960), 169173. MR $26 \# 5279$.

4. H. Lewy, An example of a smooth linear partial differential equation without solution, Ann. of Math. (2) 66 (1957), 155-158. MR 19, 551.

5. A. Martineau, Équations différentielles d'ordre infini, Bull. Soc. Math. France 95 (1967), 109-154.

6. L. Nirenberg and F. Treves, Solvability of a first order linear partial differential equation, Comm. Pure Appl. Math, 16 (1963), 331-351, MR 29 \#348.

7. - On local solvability of linear partial differential equations. Part I: Necessary conditions, Comm. Pure and Applied Math. (to appear).

8. - On local solvability of linear partial differential equations. Part II: Sufficient conditions, Comm. Pure Appl. Math. (to appear).

9. - Conditions nécessaires de résolubilité locale des équations pseudodifférentielles, C. R. Acad. Sci, Paris 269 (1969), 774-777.

10. - Conditions suffisantes de résolubilité locale des équations aux dérivées partielles linéaires, C. R. Acad. Sci. Paris 269 (1969), 853-856.

11. P. Schapira, Une équation aux dérivées partielles sans solutions dans l'espace des hyperfonctions, C. R. Acad. Sci. Paris Sér. A-B 265 (1967), A665-A667. MR 36 \#4112.

12. - Solutions hyperfunctions des équations aux dêrivées partielles du premier ordre, Bull. Soc. Math. France 97 (1969), 243-255.

13. F. Treves, $O n$ the local solvability of linear partial differential equations in two independent variables, Amer. J. Math. (to appear).

14. - Local solvability in $L^{2}$ of first order linear PDE's, Amer. J. Math. (to appear).

15. - Ovcyannikov theorem and hyperdifferential operators, Notas de Matematica, no. 46, Inst. Mat. Pura Apl. Con. Nac. Pesquisas, Rio de Janeiro, 1968.

16. - Hyperdifferential operators in complex space, Bull. Soc. Math. France 97 (1969), 193-223.

Purdue University, Lafayette, Indiana 47907 